\title{
reCHERches
}

Culture et histoire dans l'espace roman

$21 \mid 2018$

Studi per Vincenzo Consolo

\section{Vincenzo Consolo lettore di Pirandello}

\section{Cinzia Gallo}

\section{OpenEdition}

\section{Journals}

\section{Edizione digitale}

URL: https://journals.openedition.org/cher/1259

DOI: $10.4000 /$ cher.1259

ISSN: 2803-5992

\section{Editore}

Presses universitaires de Strasbourg

\section{Edizione cartacea}

Data di pubblicazione: 6 novembre 2018

Paginazione: 171-181

ISBN: 979-10-344-0027-0

ISSN: $1968-035 X$

\section{Notizia bibliografica digitale}

Cinzia Gallo, «Vincenzo Consolo lettore di Pirandello», reCHERches [Online], 21 | 2018, online dal 05 octobre 2021, consultato il 28 juin 2022. URL: http://journals.openedition.org/cher/1259 ; DOI: https:// doi.org/10.4000/cher.1259 


\title{
Vincenzo Consolo lettore di Pirandello
}

\author{
CINZIA GALL01
}

$\mathrm{L}$ attenzione e l'interesse di Consolo per Pirandello sono costanti, come dimostrano i numerosi riferimenti, espliciti od impliciti, allo scrittore agrigentino che Consolo dissemina in gran parte dei suoi lavori. La figura di Pirandello sembra intanto esemplificare la profonda influenza esercitata dai luoghi sugli individui. Infatti, se «si può cadere su questo mondo per caso, [...] non si nasce in un luogo impunemente. [...] senza essere subito segnati, nella carne, nell'anima da questo stesso luogo» (Consolo 2012: 135) ${ }^{2}$. In Uomini e paesi dello zolfo, allora, Consolo asserisce: «E, come Pirandello, ogni siciliano credo possa dire "son figlio del Caos". Ė il caos prima della formazione del cosmo, la materia informe, la "mescolanza di cose frammiste" di cui parla Empedocle (anch'egli nato nel “caos” d'Agrigento)» (Consolo 1999: 9). Ovviamente Consolo si riferisce alla grandissima varietà della terra siciliana ${ }^{3}$, dal punto di vista fisico, che gli eventi storici, però, riproducono:

Ora qui, per inciso, vogliamo notare che la storia, la storia siciliana, abbia come voluto imitare la natura: un'infinità, un campionario di razze, di civiltà sono passate per l'isola senza mai trovare tra loro amalgama, fusione, composizione, ma lasciando ognuna i suoi segni, qua e là, diversi, distinti dagli altri e in conflitto:

1 Cinzia Gallo, Università di Catania.

2 Consolo ricorda, anche, quanto Pirandello asserisce su se stesso: «Una notte di giugno caddi come una lucciola sotto un gran pino solitario in una campagna d'olivi saraceni...» (Consolo 2012: 135).

3 Sottolinea Consolo: «[...] la Sicilia, [...] quest'isola in mezzo al Mediterraneo è quanto fisicamente di più vario possa in sé raccogliere una piccola terra. Un vasto campionario di terreni, argille, lave, tufi, rocce, gessi, minerali... E quindi varietà di colture, boschi, giardini, uliveti, vigne, seminativi, pascoli, sabbie, distese desertiche. In questa terra sembra che la natura abbia subito come un arresto nella sua evoluzione, si sia come cristallizzata nel passaggio dal caos primordiale all'amalgama, all'uniformazione, alla serena ricomposizione, alla benigna quiete. Sì, crediamo che tutta la Sicilia sia rimasta per sempre quel caos fisico come quella campagna di Girgenti in cui vide la luce Pirandello» (Consolo 1999: 9). 
da qui, forse, tutto il malessere, tutta l'infelicità storica della Sicilia, il modo difficile d'essere uomo di quell'isola, e lo smarrimento del siciliano, e il suo sforzo continuo della ricerca d'identità. Ma questi problemi ci porterebbero lontano, nel magma esistenziale o nel procelloso mare pirandelliano, ed è meglio quindi che rimaniamo ancorati alla terra (Consolo 1999: 10).

Dunque, proprio perché Pirandello è «Uomo di zolfo» (Consolo 1999: 26), vissuto a stretto contatto con lo zolfo, ne tratta compiutamente nelle sue opere, che assumono carattere di denunzia. Consolo sottolinea così come nella novella Il fumo appaia chiaramente la «distruzione della campagna da parte della zolfara» (Consolo 1999: 18), mentre in Ciàula scopre la luna «la condizione del caruso [...] viene fuori in tutta la sua straziante pena; e [...] ne I vecchi e i giovani [...] il tema dello zolfo serpeggia, prima sommessamente, [...] fino ad esplodere nel finale con la rivolta degli zolfatari e con l'eccidio dell'ingegner Aurelio Costa e della sua amante [...]» (Consolo 1999: 27). Questi temi, attestanti la funzione civile della letteratura, sempre presente in Consolo, si articolano però in una filosofia «che non è sistema chiuso e definitivo, ma progressione verso [...] la poesia» (Consolo 1999: 26). Sembrerebbe, questa, una giustificazione, una spiegazione della narrazione poematica a cui Consolo approda, a partire da L'olivo e l'olivastro, anche se già ne Il sorriso dell'ignoto marinaio se ne notano delle avvisaglie.

Pirandello, «un certo Pirandello novelliere e romanziere» rappresenterebbe, inoltre, la letteratura della Sicilia occidentale, «zona fortemente implicata con la storia, [...] marcata da temi di ordine relativo - la storia, la cultura, la civiltà, la pace o la guerra sociale», mentre Verga simboleggerebbe la letteratura della Sicilia orientale, "contrassegnata [...] da temi di ordine assoluto: la vita, la morte, il mito, il fato [...]» (Consolo 2012: 134) ${ }^{4}$. È stato perciò Pirandello sottolinea Consolo - a dare ai personaggi siciliani «l'arma della dialettica, del sofisma» (Consolo 1999: 121), in sostituzione della violenza, delle passioni istintive che guidavano i contadini di Verga ${ }^{5}$. Lo spazio ristretto del villaggio di Trezza, allora, «si restringe ancora di più, si riduce alla stanza borghese, in quella che Giovanni Macchia chiama "la stanza della tortura", dove si compie ogni violenza, lacerazione, crisi, frantumazione della realtà, perdita di identità. Il movimento, in quella stanza, è solo verbale» (Consolo 1999: 269).

4 Consolo aveva espresso queste idee già nel 1986, in Sirene siciliane, considerando, però, questa «Divisione ideale, immaginaria, [...]. E questa idealità è subito contraddetta fatalmente dalla realtà, da spostamenti di autori da una parte verso l'altra: di un poeta come l'abate Meli, per esempio, verso l'Arcadia, verso la mitologia dell'Oriente, o del grande De Roberto verso la storia o lo storicismo d'Occidente» (Consolo 1999: 178-179).

5 Queste idee sono confermate nel 1999, ne Lo spazio in letteratura. Pirandello avrebbe rotto «il cerchio linguistico verghiano», portandolo «su una infinita linearità attraverso il processo verbale, la perorazione, la dialettica, i dissoi lógoi: [...] squarcia la scena con la lama dell'umorismo, trasforma l'antica tragedia nel moderno dramma» (Consolo 1999: 269). 
Non stupisce, dunque, che i due autori, Pirandello e Verga, siano posti uno di fronte all'altro ne L'olivo e l'olivastro. E non è certamente un caso che sia Pirandello, in questo testo, a rendersi conto dell'isolamento, dell'estraneità, nel suo stesso ambiente, retrivo, di Verga, estraneità che un sapiente uso dell'aggettivazione, delle figure retoriche (anafore, metafore, enumerazioni) sottolinea, costituendo, appunto, un esempio di scrittura poematica:

Pirandello lo osservò ancora e gli sembrò lontano, irraggiungibile, chiuso in un'epoca remota, irrimediabilmente tramontata. Temette che né il suo, né il saggio di Croce, né il vasto studio del giovane Russo avrebbero mai potuto cancellare l'offesa dell'insulsa critica, del mondo stupido e perduto, a quello scrittore grande, a quell'Eschilo e Leopardi della tragedia antica, del dolore, della condanna umana. Pensò che, al di là dell'esterna ricorrenza, delle formali onoranze, in quel tempo di lacerazioni, di violenza, di menzogna, in quel tramonto, in quella notte della pietà e dell'intelligenza, il paese, il mondo, avrebbe ancora e più ignorato, offeso la verità, la poesia dello scrittore. Pensò che quel presente burrascoso e incerto, sordo alla ritrazione, alla castità della parola, ebbro d'eloquio osceno, poteva essere rappresentato solo col sorriso desolato, con l'umorismo straziante, con la parola che incalza e che tortura, la rottura delle forme, delle strutture, la frantumazione delle coscienze, con l'angoscioso smarrimento, il naufragio, la perdita dell'io. Pensò che la Demente, la sua Antonietta, la suor Agata della Capinera, la povera madre, il fratello suicida di San Secondo, ogni pura fragile creatura che s'allontana, che sparisce, non è che un barlume persistente, segno di un'estrema sanità nella malattia generale, nella follia del presente (Consolo 1994: 67).

«Follia del presente» (Consolo 1994: 67) è sicuramente anche quella descritta da Consolo in gran parte dei suoi lavori: pensiamo alla realtà distorta, stravolta, frantumata propria di tutti i testi consoliani, da Il sorriso dell'ignoto marinaio a Lo Spasimo di Palermo. Giustamente, quindi, Consolo è, sostiene Anna Frabetti, un «autore di linea pirandelliana [...] in cui il sublime precipita in umoristico, il dramma borghese [...] degenera nella "vastasata", nella farsa del mondo rovesciato, privo di centro» (1995: 1). Una «Vastasata» (Consolo 1985: 10), del resto, è presente in Lunaria, in cui il Vicerè recita «la sua parte di sovrano» (Consolo 1985: 26), definisce «finzione la vita» (Consolo 1985: 66), si mostra consapevole, ricorrendo ad interrogative retoriche ed enumerazioni, della vanità, del carattere relativo del reale: «Dov'è Abacena, Apollonia, Agatirno, Entella, Ibla, Selinunte? Dov'è Ninive, Tebe, Babilonia, Menfi, Persepoli, Palmira? Tutto è maceria, sabbia, polvere, erbe e arbusti ch'hanno coperto i loro resti» (Consolo 1985: 61); e Tutti commentano, con sapiente uso delle figure retoriche: "Così è stato e così [anafora] sempre sarà [poliptoto]: rovinano potenze, tramontano imperi regni civiltà [enumerazione], cadono astri, si sfaldano, si spengono [climax], uguale sorte hanno mitologie credenze religioni. Ogni fine è dolore, smarrimento ogni mutazione [chiasmo], stiamo saldi, pazienza, in altri teatri, su nuove illusioni nascono certezze» (Consolo 1985: 34). E la Sesta donna: «Tutto si frantuma, / cade, passa [climax]» (Consolo 1985: 54). 
Su questa scia, nella prima sezione di Retablo, Isidoro scorge, nella chiesa di S. Lorenzo, una statua, che reca sul piedistallo la parola«VERITAS» (Consolo 1992: 19), dalle fattezze simili a quelle di Rosalia, ad attestare il carattere apparente, relativo del reale. Lo stesso significato ha, nella terza sezione, l'espressione «Bella, la verità» (Consolo 1992: 149) ${ }^{6}$ ripetuta da Rosalia che, del resto, sottolinea esplicitamente il contrasto fra apparenza e realtà: «Bagascia, sì, all'apparenza, ma per il bene nostro, tuo e mio»; "Fu per questo che scappai, ch'accettai questa parte dell'amante, questa figura della mantenuta» (Consolo 1992: 149, 155).

Se pirandelliana è la costrizione dell'individuo in una forma, Pirandello offre pure le coordinate con cui spiegare l'aspirazione ad essere diversi da quello che si è e con cui si ritiene che ciò sia possibile modificando l'aspetto esteriore, la propria forma, a svuotare di consistenza ruoli e funzioni. Ecco che Consolo, ne Le vele apparivano a Mozia, ricorda come l' «autista-inserviente-guardiano» del pittore Guttuso ${ }^{7}$, «dal bel nome greco dalla Spagna poi donato alla Sicilia d'Isidoro $^{8}[\ldots]$ come nella novella di Pirandello Sua Maestà, in un desiderio di mimesi, di immedesimazione, si vestiva alla stessa maniera del padrone: giacca e pantaloni blu, camicia azzurra, pullover rosso, fazzoletto rosso che trabocca dal taschino» (Consolo 2012: 124). Anche l'importanza data ai nomi si pone, del resto, sulla scia di Pirandello, che - è noto - istituisce uno stretto «rapporto» fra «nome - identità dei personaggi» (De Villi2013: 278), "per affinità o per antifrasi» (De Villi 2013: 277). Analogamente Consolo esclama: «il destino dei nomi!» (Consolo 2012: 128) ${ }^{9}$.

E la parola, il nome è spesso segno di predestinazione o di destino. Dei nomi dati agli uomini, voglio dire, e dei destini degli uomini: il destino dei nomi. Ma non sappiamo se è l'uomo sul nascere, già segnato da un destino, che si versa e assesta dentro il suo giusto e appropriato involucro di nome (e cognome) oppure se sono il nome e il cognome che, capitati per caso sulla pelle di un uomo come maglietta e brache, ne incidono le carni, ne determinano cioè il destino (Consolo 2012: 66).

Definisce, così, le poesie della poetessa Assunta Della Musa, «fra le più ispirate, le più eccitate, le più squisite e belle tra le poesie d'amore scritte in tutti i tempi e in tutti i luoghi. [...] Può una donna di nome Assunta Della Musa, coniugata ad Apollo Barilà, non scrivere poesie, essere della poesia, essere la poesia? Essere Erato, la poesia erotica?» (Consolo 2012: 69). Perciò, non a caso, con chiara allusione al leopardiano Dialogo di Plotino e Porfirio, in cui quest'ultimo è

6 Cfr., su questo argomento, Galvagno 2015: 39-64.

7 E, questi, l'unico pittore a cui Consolo attribuisce, ne L'enorme realtà, «il dono della capacità del racconto, della rappresentazione [...] che hanno avuto scrittori come Verga, come Pirandello, come Sciascia» (Consolo 1999: 271).

8 Con caratteristiche simili, a confermare l'importanza dei nomi, in Lunaria si chiama Isidoro il maestro di cerimonie del Vicerè, attento alle apparenze, «intransigente custode di $[\ldots]$ inderogabili forme palatine» (16).

9 In Lunaria, gli abitanti della «selvaggia Contrada senza nome» sono «uomini senza legge, senza lingua, senza storia, anime boschive, $[\ldots] »(61)$. 
consapevole della «vanità di ogni cosa» (Leopardi 1978: 530), si chiama Porfirio il valletto di Casimiro, il vicerè di Lunaria, che non prende mai la parola, ma è consapevole della «recitazione» (Consolo 1985: 10) del suo signore.

Lucia, poi, si chiama - per antifrasi in rapporto all'etimologia del nome -, la sorella di Petro Marano, affetta da disturbi mentali. E il quinto capitolo di Nottetempo, casa per casa, che la mostra, alla fine, pazza, reca, in epigrafe, una battuta di Come tu mi vuoi: "Chiami, chi sa da qual momento lontano... felice.../ della tua vita, a cui sei rimasta sospesa... là...» (Consolo 1992: 59). Erasmo, ancora, con probabile riferimento ad Erasmo da Rotterdam e al suo Elogio della follia - oltre che, a confermare la rilevanza attribuita dal nostro scrittore allo spazio - al piano di sant'Erasmo, nei dintorni di Palermo, si chiama il «vecchietto lindo, bizzarro» (Consolo 1998: 103) de Lo Spasimo di Palermo, a cui è affidato il compito di mettere in evidenza, alla fine del romanzo, l'importanza della letteratura. Costui, infatti, coinvolto nell'attentato al giudice Borsellino, recita, in punto di morte, due versi de La storia di la Baronissa di Carini, ad attestare come, anche se al presente la letteratura non è ascoltata, è da questa, voce della tradizione, della memoria storica, che deve venire la salvezza:

O gran mano di Diu, ca tantu pisi,

cala, manu di Diu, fatti palisi! (Consolo 1998: 131)

E, ancora, Consolo dichiara: «La salvezza è stata solo nel linguaggio. Nella capacità di liberare il mondo dal suo caos, di rinominarlo, ricrearlo in un ordine di necessità e di ragione» (Consolo 1999: 272). Petro Marano, perciò, si aggrappa «alla parole, ai nomi di cose vere, visibili, concrete», desideroso di «rinominare, ricreare il mondo» (Consolo 1992: 42-43). Egli, poi, alla fine si rifugia a Tunisi, così come anche Lando Laurentano avrebbe voluto imbarcarsi per Malta o per Tunisi (Pirandello 1953: 394).

Consolo, quindi, mette in relazione, attraverso la figura di Antonio Crisafi de La pallottola in testa, il disagio, l'estraneità dell'intellettuale nella moderna società, sia quella del «Meridione depresso» (Consolo 2012: 157) sia quella legata all'avvento dei mass media, della televisione, all'isolamento del professor Lamis de L'eresia catara di Pirandello. Ed anche in Un giorno come gli altri, discutendo della funzione dell'intellettuale, Consolo si richiama a Pirandello. A proposito, infatti, della differenza, instaurata da Moravia e Vittorini, fra artista e intellettuale, egli asserisce:

A me la distinzione sembra vecchia, mi ricorda l'affermazione di Pirandello: "La vita, o la si scrive o la si vive”. Ché l'alternativa, oltre a valere per tutti, non solo per l'artista, dopo Marx non ha più senso. Oggi siamo tutti intellettuali, siamo tutti politici, [...]. Il problema mi sembra che stia nel voler essere o no dentro le "regole", nel voler essere o no, totalmente, incondizionatamente, dentro un partito, dentro la logica "politica" di un partito. Questo mi sembra il punto, il punto di Vittorini (Consolo 2012: 91-92).

Arriva, quindi, alla sua celebre distinzione fra scrivere e narrare:

Riprendo a lavorare a un articolo per un rotocalco sul poeta Lucio Piccolo. Mi accorgo che l'articolo mi è diventato racconto, che più che parlare di Piccolo [...] 
in termini razionali, critici, parlo di me, della mia adolescenza in Sicilia, di mio nonno, del mio paese: mi sono lasciato prendere la mano dall'onda piacevole del ricordo, della memoria. [...] È [...] il narrare, operazione che attinge quasi sempre alla memoria, [...]. Diverso è lo scrivere, [...] operazione $[. .$.$] impoetica,$ estranea alla memoria, che è madre della poesia, come si dice. E allora è questo il dilemma, se bisogna scrivere o narrare. Con lo scrivere si può forse cambiare il mondo, con il narrare non si può, perché il narrare è rappresentare il mondo, cioè ricrearne un altro sulla carta (Consolo 2012: 92) ${ }^{10}$.

Pirandello simboleggia la Sicilia, insieme a Verga, Meli, Capuana, secondo il mafioso catanese, sottoposto al 41 bis nel carcere di Opera - Milano, dopo aver «fatto un bel po' di strada negli affari, appalti, commerci vari» (Consolo 2012: 216): Consolo ironizza sulla politica separatista, portata avanti dal Movimento indipendentista siciliano di Finocchiaro Aprile e, di conseguenza, sulla politica della Lega Nord, sottolineando ancora una volta l'importanza della memoria storica.

Si pone, ancora, accanto a Pirandello, dichiarando che $\mathrm{Il}$ sorriso dell'ignoto marinaio "era d'impianto storico» ma «voleva anche dire metaforicamente del momento che allora si viveva, a Milano e altrove» (Consolo 2012: 119): «(si svolgeva negli anni del Risorgimento e dell'impresa garibaldina: nodo di passaggio storico importante per il Meridione e banco di prova della maggior parte degli scrittori siciliani - Verga, De Roberto, Pirandello, Lampedusa, Sciascia...)» (Consolo 2012: 119) ${ }^{11}$, accomunati tutti, «da Verga a De Roberto, a Pirandello», da «un costante immobilismo» (Consolo 1999: 169)12, pur nella diversità delle posizioni ideologiche. In particolare, se la rinuncia a rappresentare la rivolta parrebbe accomunare Il sorriso dell'ignoto marinaio a I vecchi e i giovani, le motivazioni dei due scrittori sono differenti. Consolo, consapevole «dei limiti di classe degli intellettuali», nutre «sfiducia nella possibilità, da parte della letteratura, di rendere la visione e il sentire delle classi subalterne senza stravolgimenti mistificatori»; Pirandello, invece, è mosso da un profondo "pessimismo» che lo induce «a svalutare anche gli eventi più tragici ed epocali come frutto di vane illusioni e follie destinate ad essere cancellate dal

10 Nel 1997, richiamandosi alle tesi espresse da Walter Benjamin in Angelus novus, Consolo preciserà: «E c'è, nella narrazione, un'idea pratica di giustezza e di giustizia, un'esigenza di moralità». (Consolo 1999: 144). Per quest'argomento, cfr. Francese 2015. L'influsso di Benjamin su Consolo è stato evidenziato anche da Daragh O'Connell (2008: 161-184), che ricorda la traduzione in italiano de Il narratore di Benjamin effettuata, per Einaudi, da Renato Solmi nel 1962 (162, nota 2).

11 Pure ne Il sorriso, vent'anni dopo, Consolo asserisce che il suo romanzo è nato da una «rilettura della letteratura che investe il Risorgimento, soprattutto siciliana, ch'era sempre critica, antirisorgimentale, che partiva da Verga e, per De Roberto e Pirandello, arrivava allo Sciascia de Il Quarantotto, fino al Lampedusa de $I l$ Gattopardo» (Consolo 1999: 279).

12 Consolo ricorda come i critici di orientamento lukácsiano avessero posto Il Gattopardo accanto a I Vicerè di De Roberto e a I vecchi e i giovani di Pirandello (Consolo 1999: 173). 
tempo, [...]» (Baldi 2014: 254). In entrambi i romanzi, però, il Risorgimento si risolve in una «disillusione del vecchio sogno della terra» (Consolo 2012: 109) ${ }^{13}$ e nei pensieri di Lando Laurentano si scorge un'eco di quei contrasti di classe che Consolo pone in primo piano ${ }^{14}$ : «Da una parte il costume feudale, l'uso di trattar come bestie i contadini, e l'avarizia e l'usura; dall'altra l'odio inveterato e feroce contro i signori e la sconfidenza assoluta nella giustizia, si paravano come ostacoli insormontabili a ogni tentativo per quella cooperazione» (Pirandello 1953: 392 ${ }^{15}$. E precedentemente, ascoltando il discorso di Cataldo Sclàfani, considera: «Una buona legge agraria, una lieve riforma dei patti colonici, un lieve miglioramento dei magri salarii, la mezzadria a oneste condizioni, come quelle della Toscana e della Lombardia, come quelle accordate da lui nei suoi possedimenti, sarebbero bastati a soddisfare e a quietare quei miseri [...]» (Pirandello 1953: 288).

$\mathrm{Ne}$ Il sorriso dell'ignoto marinaio, del resto, anche la figura di Garibaldi, su cui si concentrano le aspettative dei 'giovani' (Roberto Auriti, Mauro Mortara, Corrado Selmi, Rosario Trigona), consente di stabilire delle corrispondenze con Pirandello. Consolo, difatti, sottolinea il favore ottenuto da Garibaldi («[...] vanno dicendo che [Garibaldi] gli dà giustizia e terre.»), ritenuto però, al tempo stesso, un «Brigante. Nemico di Dio e di Sua Maestà il Re [...] Scanna monache e brucia conventi, rapina chiese, preda i galantuomini e protegge avanzi di galera...» (Consolo 2004: 66).

E ribadisce le sue riserve su Garibaldi anche in altri testi. Parlando, nel 1982, della rivolta di Bronte, dell'agosto 1860, Consolo, oltre ad evidenziare la «crudeltà», la «sommarietà di giustizia» (Consolo 2012: 108) di Bixio, afferma riguardo Garibaldi:

In questa annata di celebrazione garibaldinesca in chiave post-moderna, in cui tutti gli stili, le citazioni, i repêchages si fanno stile, in cui le pagine chiare e oscure, le glorie e le vergogne, le vittime e gli scheletri, più che nascosti nell'armadio, esibiti si fanno levigato stile eroico, gloriosa epopea da consumo, soffermarsi

13 Scrive Pirandello: «Sì, aveva esposto la verità dei fatti quel deputato siciliano: quei contadini di Sicilia, [...] s'erano recati a zappare le terre demaniali usurpate dai maggiorenti del paese, amministratori ladri dei beni patrimoniali del Comune: intimoriti dall'intervento dei soldati, avevano sospeso il lavoro ed erano accorsi a reclamare al Municipio la divisione di quelle terre; [...]» (Pirandello 1953: 238).

14 Pensiamo a quest'episodio, che trova corrispondenza nella terza scritta al nono capitolo: "Ah ah, puzzo di merda, papà, ah ah" sentirono ancora alle spalle che faceva Salvatorino, grasso come 'na femmina, babbalèo, mammolino, ancora a quindici anni sempre col dito in bocca, la bava e il moccio, unico erede, presciutto tesoro calasia, al padre professore Ignazio e al nonno sindaco, il notaio Bàrtolo. / Tanticchia girò la testa sopra il tronco e lo guatò sbieco. / "Garrusello e figlio di garruso alletterato!" disse, e poi sputò per terra, bianco e sodo, tondo come un'onza» (Consolo 2004: 95-96).

15 Lo stesso Consolo ricorda le «Insurrezioni che spesso non sono solo contro i borbonici, ma di contadini e braccianti contro i loro nemici di sempre, i nobili e i borghesi che quasi dappertutto avevano usurpato terre demaniali» (Consolo 2012: 107). 
su un episodio come quello di Bronte, estrapolarlo dal contesto post-moderno, appunto, può farci apparire fuori moda, arretrati, forse striduli (Consolo 2012: 107).

Ma che Consolo consideri in modo non del tutto positivo Garibaldi e il suo influsso è dimostrato, ancora, dai giudizi formulati nell'articolo Il più bel monumento:

Questo ironico (speriamo) e autoironico personaggio, nella sua campagna d'Italia, non fece che imitare, nel dire, nel fare e nel posare, il monumento di sé ch'era già idealmente eretto, in uno spassoso scambio tra l'immagine e il reale, in gara di esaltazione e in doppio accrescimento senza fine. Tutti rimasero vittime del giuoco, e ogni città e villa non poté che innalzargli il monumento. [...] Ed era questo che Garibaldi in fondo desiderava: volare, volare in un teatrino d'invenzione per dimenticare le colpe e sopire i rimorsi che dentro gli rodevano (Consolo 2012: 70-71).

Analogamente, nella novella pirandelliana L'altro figlio, Garibaldi è colui che «fece ribellare a ogni legge degli uomini e di Dio campagne e città» (Pirandello 1955: 242). E Maragrazia prosegue, servendosi di enumerazioni, metafore, esclamazioni, paragoni, puntini di sospensione, per coinvolgere emotivamente il lettore e rendere il suo racconto più persuasivo:

[...] vossignoria deve sapere che questo Canebardo diede ordine, quando venne, che fossero aperte tutte le carceri di tutti i paesi. Ora, si figuri vossignoria che ira di Dio si scatenò allora per le nostre campagne! I peggiori ladri, i peggiori assassini, bestie selvagge, sanguinarie, arrabbiate da tanti anni di catena... Tra gli altri, ce n'era uno, il più feroce, un certo Cola Camizzi, capo-brigante, che ammazzava le povere creature di Dio, così, per piacere, come se fossero mosche, per provare la polvere, - diceva - per vedere se la carabina era parata bene. [...] Ah, che vidi! [...] Giocavano... là, in quel cortile... alle bocce... ma con teste d'uomini... nere, piene di terra... le tenevano acciuffate pei capelli... e una, quella di mio marito... la teneva lui, Cola Camizzi... e me la mostrò. [...] cane assassino! (Pirandello 1955: 242-244).

Pirandello è per Consolo, ancora, il termine di paragone attraverso cui giudicare i testi della contemporaneità, a metterne in evidenza la vitalità, il carattere paradigmatico. Asserisce così: "La storia di Creatura di sabbia [di Tahar Ben Jelloun] è una delle più felici invenzioni letterarie del romanzo contemporaneo, uguale forse, per la metafora, per la verità profonda che riesce a liberare, a quella de Il fu Mattia Pascal di Pirandello» (Consolo 1999: 232-233). Analogamente, pure vari aspetti della produzione di Sciascia sono spiegati in rapporto a Pirandello. Nella prefazione a Le epigrafi di Leonardo Sciascia di Pino Di Silvestro, Consolo considera quale «più grande epigrafe di tutta l'opera di Sciascia, non scritta ma vistosamente implicita, [...] la stanza della tortura pirandelliana declinata sul piano della storia, sul palcoscenico della violenza, della sconfitta» (Consolo 1999: 202). Tre anni più tardi, nel 1999, Consolo, evidenziando la funzione civile sottesa all'opera di Sciascia, gli attribuisce il merito di avere spostato «la dialettica pirandelliana dalla stanza alla piazza, nella civile agorà» (Consolo 1999: 269). Sciascia, allora, in questa sua «conversazione 
loica e laica sui fatti sociali e politici» si rivela "figlio di Pirandello» (Consolo 1999: 186), al punto tale che il personaggio narrante di Todo modo è «nato e per anni vissuto in luoghi pirandelliani, tra personaggi pirandelliani - al punto [dice] che tra le pagine dello scrittore e la vita che avevo vissuto fin oltre la giovinezza, non c'era più scarto, e nella memoria e nei sentimenti» (Consolo 1999: 187-188).

Quest'interesse, questa consonanza di idee con Pirandello, porta Consolo a riunire in Di qua dal faro, con il titolo di Asterischi su Pirandello, alcuni saggi dedicati allo scrittore agrigentino, pubblicati fra il 1986 e il 1997. In Album Pirandello Consolo ribadisce la funzione modellizzante che lo spazio ha esercitato su tutta la famiglia dello scrittore agrigentino: «quell'albero genealogico [...] dispiega i suoi rami contro un cielo di luce crudele, affonda le sue radici in quell'asperrimo terreno che è la Sicilia, in quel caos di marne e di zolfi che è Girgenti» (Consolo 1999: 150). E così anche l'eclissi di sole a cui assistette "graverà sul mondo dello scrittore» (Consolo 1999: 150) e si combinerà con "quella [...] della città in cui si trovò a vivere, di Girgenti. Una città dove è morta la storia, la civiltà, lasciando il vuoto, il deserto, [...] la stasi, l'immobilità» (Consolo 1999: 150-151). Ricordiamo, difatti, che Consolo, ne L'olivo e l'olivastro e ne Lo Spasimo di Palermo, per esempio, individua, nella perdita della memoria storica, la causa della crisi del presente. Scrive così: «si può mai narrare senza la memoria?»; "Non è vero, io non so scrivere di Milano, non ho memoria» (Consolo 2012: 88, 97).

Unica soluzione, allora, l'evasione, come quelle di Mattia Pascal o di Enrico IV, oppure rivestire delle forme, difenderle con le armi della dialettica, del sofisma, della retorica. L'operazione di Pirandello sembra perciò trovare dei riscontri nell'età contemporanea, in cui «l'io s'è perso nell'indistinta massa, la vita nelle prigioni sempre più disumane delle forme imposte dal potere, l'essere nell'apparire fantasmatico dei media» (Consolo 1999: 152). E non dimentichiamo che pure Consolo considera negativamente l'omologazione. Gioacchino Martinez, per esempio, sul treno che lo conduce a Palermo, prova piacere «a risentire quei suoni, quelle cadenze meridionali, quelle parlate che non erano più dialetto, ma non ancora la trucida nuova lingua nazionale» (Consolo 1998: 94-95) annunciata da Pasolini. Il viaggiatore de L'olivo e l'olivastro, poi, giudica «vacui» i giovani che, «con l'orecchino al lobo, i lunghi capelli legati sulla nuca» (Consolo 1994: 112), affollano la piazza di Avola.

Altri legami fra Pirandello e Consolo ne L'ulivo e la giara. Gli stucchi di Giacomo Serpotta, che lo scrittore agrigentino ebbe modo, molto probabilmente, di osservare nella chiesa di Santo Spirito, con il loro carattere «mortuario [...] fantasmatico» che "ha colto il pittore Fabrizio Clerici nella sua Confessione palermitana» (Consolo 1999: 156), hanno influenzato pure Consolo, il quale, in Retablo, chiama Fabrizio Clerici il protagonista e descrive le sculture in stucco dell'oratorio di via Immacolatella di Procopio Serpotta, figlio di Giacomo. «La bianca, spettrale fantasmagoria serpottiana» (Consolo 1999: 156), inoltre, richiama la «servetta Fantasia» attraverso cui i vari personaggi delle opere 
letterarie si materializzano, così come Macchia per Pirandello e Carandente per Serpotta parlano del «cannocchiale rovesciato» (Consolo 1999: 157). Analogamente, la superiorità di Cefalù su Palermo, sostenuta da Consolo varie volte $^{16}$, è colta anche da Pirandello. Consolo immagina che questi, in viaggio da Palermo a Sant'Agata, in preda alla profonda suggestione "che gli suscitavano i nomi dei paesi: Solunto, Himera, Cefalù, Halaesa, Calacte...», si accorge che, «dopo Cefalù, il mondo colorato, vociante e brulicante del Palermitano andava a poco a poco stemperandosi [per] a prendere gradualmente una misura più dimessa, ma forse più serena» (Consolo 1999: 157-158).

Pirandello, a confermare l'importanza dei luoghi, ebbe sicuramente presente, secondo Consolo, «il ricordo di quel suo lontano viaggio nel Val Dèmone» (Consolo 1999: 161) nello scrivere La giara, «la prima fuga nella memoria e nel ricordo, fuga dalla sua vita e dai fantasmi "pirandelliani" che lo assediavano» (Consolo 1999: 160). La novella, perciò, giudicata di recente una «divertita denuncia dell'intrinseca capziosità sia delle vicende che delle soluzioni giuridiche, calata in pieghe di umoristica densità» (Zappulla Muscarà 2007: 143), acquista nuovo significato nell'interpretazione di Consolo. La giara è per lui, infatti, a richiamare la sua tipica figura chiave della chiocciola, della spirale, sia «l'involucro della nascita, l'utero» sia «la tomba», mentre «quell'olio che la giara avrebbe dovuto contenere viene sì dall'ulivo saraceno, ma viene anche dall'albero sacro ad Atena, dea della sapienza» (Consolo 1999: 161-162), a ricordare la commistione delle due culture, araba e greca, della Sicilia. Consolo può allora vedere nel pino di Pirandello, tranciato, un simbolo degli «scadimenti, delle perdite, reali e simboliche, nel nostro Paese» (Consolo 1999: 163), a confermare la «visione del mondo, della vita come caos, mutamento incessante di forme, [...] approdo all'assenza, al nulla» (Consolo 1999: 165). Anche in ciò Consolo si trova in consonanza con Sciascia ${ }^{17}$, che commenta, alla fine di Fuoco dell'anima: "Questa è la classe dirigente - per meglio dire digerente - che preferisce fare il pino di plastica piuttosto che salvare quello vero. Ed è così per tante, tante altre cose...» (Consolo 1999: 164).

Con tutto questo, Consolo mostra l'importanza della ricezione dei testi letterari, avvicinandosi al lector in fabula descritto da Eco (1979).

16 Mi sia consentito, per questo, un rimando a Gallo 2017: 287-296.

17 Gianni Turchetta sottolinea, a proposito del termine 'impostura' de Il sorriso dell'ignoto marinaio, i legami di Consolo con Sciascia (2015: 1304-1305). Ne Lo Spasimo di Palermo, inoltre, Gioacchino Martinez legge, ne La corda pazza, la vita di Antonio Veneziano (115) e il narratore ricorda il «rifugio in Solferino dove Sciascia patì la malattia, sua del corpo e insieme quella mortale del Paese» (93-94). 


\section{Bibliografia}

Baldi G., 2014, Microscopie, Napoli, Liguori.

Consolo V., 1985, Lunaria, Torino, Einaudi.

Consolo V., 1992, Nottetempo, casa per casa, Milano, Mondadori.

Consolo V., 1992, Retablo, Milano, Mondadori.

Consolo V., 1994, L'olivo e l'olivastro, Milano, Mondadori.

Consolo V., 1998, Lo Spasimo di Palermo, Milano, Mondadori.

Consolo V., 1999, Di qua dal faro, Milano, Mondadori.

Consolo V., 2004, Il sorriso dell'ignoto marinaio, Milano, Mondadori.

Consolo V., 2012, La mia isola è Las Vegas, Milano, Mondadori.

Consolo V., 2015, L'opera completa, a cura e con un saggio introduttivo di Gianni Turchetta e uno scritto di Cesare Serge, Milano, Mondadori, «I Meridiani».

De Villi A. I., 2013, "Pasquale Marzano, Quando il nome è "cosa seria". L'onomastica nelle novelle di Luigi Pirandello», (Pisa, ETS, 2008, 206 p.), OBLIO III, 9-10, p. 277-279.

Eco U., 1979, Lector in fabula, Milano, Bompiani.

Frabetti A., 1995, “L”infinita derivanza”. Intertestualità e parodia in Vincenzo Consolo», Bollettino '900, n. zero, maggio, n. 1, http://www.comune.bologna. it/iperbole/boll900/consolo.htm

Francese J., 2015, Vincenzo Consolo, Firenze, University Press.

Gallo C., 2017, La Yoknapatawpha di Vincenzo Consolo, in: Sgavicchia S., Tortora M., Geografie della modernità letteraria, Pisa, ETS, I, p. 287-296.

Galvagno R., 2015, “Bella, la verità”. Figure della verità in alcuni testi di Vincenzo Consolo,» in: Diverso è lo scrivere. Scrittura poetica dell'impegno in Vincenzo Consolo, Avellino, Edizioni Sinestesie, p. 39-64.

Leopardi G., 1978, I Canti. Operette morali, Roma, Casa Editrice Bietti.

O'Connell D., 2008, "Consolo narratore e scrittore palincestuoso», Quaderns d'Italià, 13, p. 161-184.

Pirandello L., 1953, I vecchi e i giovani, in: Tutti i romanzi, vol. II, Milano, Mondadori.

Pirandello L., 1955, Novelle per un anno, Milano, Mondadori, vol. II.

Turchetta G., 2015, «Note e notizie sui testi», in: Consolo V., L'opera completa, p. 1271-1455.

Zappulla Muscarà S., 2007, «La Giara e La patente fra narrativa e teatro ovvero Pirandello nell'isola del sofisma», in: Lauretta E., Novella di Pirandello: dramma, film, musica, fumetto, Pesaro, Metauro, p. 143-167. 